\title{
ON KNOWLEDGE DISCOVERY AND REPRESENTATIONS OF MOLECULAR STRUCTURES USING TOPOLOGICAL INDICES
}

\author{
Fawaz E. Alsaadi ${ }^{1}$, Syed Ahtsham Ul Haq Bokhary ${ }^{2}$, Aqsa Shah ${ }^{2}$, \\ Usman $\mathrm{Ali}^{2}$, Jinde $\mathrm{Cao}^{3}$, Madini Obad Alassafi ${ }^{1}$, \\ Masood Ur Rehman*,4, Jamshaid Ul Rahman ${ }^{5}$ \\ ${ }^{1}$ Department of Information Technology, Faculty of Computing and Information Technology, \\ King Abdulaziz University, Jeddah21589, Saudi Arabia \\ ${ }^{2}$ Centre of Advanced Studies in Pure and applied Mathematics, \\ Bahauddin Zakariya University, Multan, Pakistan \\ ${ }^{3}$ School of Mathematics, Southeast University, Nanjing 210096, China \\ ${ }^{4}$ Department of Mathematics, Abbottabad University of Science and Technology, \\ Abbottabad, 22500, Pakistan \\ ${ }^{5}$ School of Mathematical Science, University of Science and Technology of China, \\ Hefei 230026, Anhui, P.R. China \\ *E-mail: masoodqau27@gmail.com
}

Submitted: 11th March 2020; Accepted: 25th August 2020

\begin{abstract}
The main purpose of a topological index is to encode a chemical structure by a number. A topological index is a graph invariant, which decribes the topology of the graph and remains constant under a graph automorphism. Topological indices play a wide role in the study of QSAR (quantitative structure-activity relationship) and QSPR (quantitative structure-property relationship). Topological indices are implemented to judge the bioactivity of chemical compounds. In this article, we compute the $A B C$ (atom-bond connectivity); $A B C_{4}$ (fourth version of $A B C$ ), $G A$ (geometric arithmetic) and $G A_{5}$ (fifth version of $G A$ ) indices of some networks sheet. These networks include: octonano window sheet; equilateral triangular tetra sheet; rectangular sheet; and rectangular tetra sheet networks.
\end{abstract}

Keywords: graph network, sheet, topological index, cheminformatics, knowledge discovery

\section{Introduction and preliminary re- sults}

In chemical graph theory, an interesting subfield called Cheminformatics deals with a chemical phenomenon known as quantitative structureactivity/ structure- property relationships of chem- ical compounds. The methods of cheminformatics are also used for prediction of properties relevant to the drug discovery and optimization process. For example,knowledge discoverycan be used for the identification of lead compounds in pharmaceutical data matching. An emerging tool, used in the study of these phenomena, is a topological index 
which remains constant for all chemical structures up to their symmetries. The study of the topological indices on chemical structure drugs can provide a theoretical basis for the manufacturing of drugs and chemical materials. As a consequence, lack of chemical experiments is made up. A number of topological indices are determined in view of edge dividing methods which provide remedy to the lack of medicine experiments. In other words, computation of topological indices provides a theoretical basis for pharmaceutical engineering. Correlation of many physico-chemical properties like boiling point; stability; and strain energy of these chemical compounds in a chemical structure is explained by their topological indices $[2,7,17,19,20]$.

In this era of rapid technological development, chemical and pharmaceutical techniques in recent years have been rapidly evolved, and thus a large number of new nanomaterials, crystalline materials, and drugs emerge every year. To determine the chemical properties of such a large number of new compounds and new drugs requires a large amount of chemical experiments, thereby greatly increasing the workload of the chemical and pharmaceutical researchers. Fortunately, the chemical based experiments found that there was strong connection between topology molecular structures and their physical behaviors, chemical characteristics, and biological features, such as melting point, boiling point, and toxicity of drugs (see Wiener [24] as examples).

The description of a graph can be a number; a polynomial; a sequence of numbers; or a matrix. A numerical quantity related to a graph that represents the topology of the graph is a topological index. There are several main types of topological indices such as distance based topological indices and degree based topological indice. Among these indices, a degree-based topological index is very important and plays a vital role in chemical graph theory. More precisely, a topological index $\operatorname{Top}(G)$ of a graph $G$ is a number that remains the for every graph $H$ such that $H \cong G$. In other words, we have $\operatorname{Top}(H)-\operatorname{Top}(G)=0$. In [24], Wiener introduced the first topological index while working on the boiling point of paraffin. He named this index the path number. After a period of time, the path number was renamed Wiener index in [5] and the theory of topological indices got attention from many researchers.
In present work, by a graph $G$ we always mean a network with vertex set $V(G)$ and edge set $E(G)$. We denote the degree of a vertex $u$ of $G$ by $\operatorname{deg}(u)$ (degree of a vertex $u$ of a graph is the number of edges that are incident to the vertex $u$ ) and $S_{u}$ is the sum of degrees of vertex $v$ which is the in neighbour of vertex $u$ i.e., $S_{u}=\sum_{v \in N_{G}(u)} \operatorname{deg}(v)$, where $N_{G}(u)$ is the of vretices which are in the neighbours of vertex $u$, i.e., $N_{G}(u)=v \in V(G) \mid u v \in E(G)$.

The atom-bond connectivity $(A B C)$ is the wellknown degree based topological index, which is introduced by Estrada et al. [7] and defined as

$$
A B C(G)=\sum_{u v \in E(G)} \sqrt{\frac{\operatorname{deg}(u)+\operatorname{deg}(v)-2}{\operatorname{deg}(u) \operatorname{deg}(v)}} .
$$

The geometric-arithmetic $(G A)$ index is due to Vukičevic' et al. [23] and defined as

$$
G A(G)=\sum_{u v \in E(G)} \frac{2 \sqrt{\operatorname{deg}(u) \operatorname{deg}(v)}}{(\operatorname{deg}(u)+\operatorname{deg}(v))} .
$$

Ghorbani et al. [9] introduced the fourth version of $A B C$ index denoted by $A B C_{4}(G)$ and defined as

$$
A B C_{4}(G)=\sum_{u v \in E(G)} \sqrt{\frac{S_{u}+S_{v}-2}{S_{u} S_{v}}} .
$$

Recently, the fifth version of $G A$ index is proposed by Graovac et al. [10] and defined as

$$
G A_{5}(G)=\sum_{u v \in E(G)} \frac{2 \sqrt{S_{u} S_{v}}}{\left(S_{u}+S_{v}\right)} .
$$

The aforementioned topological invariants are hugely studied in literature. Some of the work done in this direction can be found in [1-24].

In present work, we study the $A B C, A B C_{4}$, $G A$, and $G A_{5}$ indices of certain networks sheets including octo-nanowindows sheet; enhanced mesh or rectangular sheets; HDN like networks namely equilateral triangular tetra sheets; and rectangular tetra sheets networks.

\section{Octo - nanowindows network sheet}

In this Section, we are in the position to compute $A B C, A B C_{4}, G A$, and $G A_{5}$ indices of octonanowindow sheet networks. 


\subsection{Construction of octo - nanowindow sheet}

First we draw an octagon where the vertices are the eight corners and an edge links these corners with a length of 2 units. We paste the $p$ octagons row wise and $q$ octagons column wise to get a graph $G$ which is known as octo-sheet. Connect the degree 2 vertices of each octagon which are at distance 4 units. Finally, introduce 4 new corner vertices and connect them to the corner vertices of the corner octagon with a new edge of unit 1. The graph obtained in this way is called octonanowindow $O N W(4 p, 4 q)$ having $2(2 p q+p+q+$ $2)$ vertices and $6 p q+3 p+3 q+4$ edges. The graph of $O N W(4 p, 4 q)$ is shown in Figure 1.

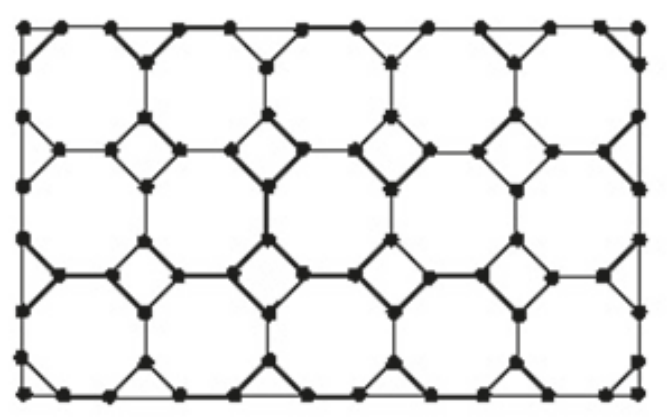

Figure 1. The octo-nanowindo $O N W(4 p, 4 q)$ network, where $p=3$ and $q=5$.

Table 1. Edge partition of $O N W(4 p, 4 q)$ which depends on the degrees of the final vertices located at unit distance from each edge.

\begin{tabular}{|c|c|}
\hline$\left(d_{u}, d_{v}\right)$ where $u v \in E(G)$ & Number of Edges \\
\hline$(2,3)$ & 8 \\
\hline$(3,3)$ & $6 p q+3 p+3 q-4$ \\
\hline
\end{tabular}

The following theorem gives the $A B C$ and $G A$ indices of octo-nanowindow $O N W(4 p, 4 q)$.

Theorem 2.1 Suppose that $G$ is the graph of octonanowindow $O N W(4 p, 4 q)$. Then, $A B C(G)=$ $4 p q+2 p+2 q+\left(4 \sqrt{2}-\frac{8}{3}\right)$, for $p, q>1$. Proof. By following the data given in Table 1, we have

$A B C(G)=\sum_{u v \in E(G)} \sqrt{\frac{d_{u}+d_{v}-2}{d_{u} d_{v}}}=8 \sqrt{\frac{2+3-2}{2 \times 3}}+(6 p q+$ $3 p+3 q-4) \sqrt{\frac{3+3-2}{3 \times 3}}$. After an easy calculation, we get $A B C(G)=4 p q+2 p+2 q+\left(4 \sqrt{2}-\frac{8}{3}\right)$.

Theorem 2.2 With the same notations, we have
$G A(G)=6 p q+3 p+3 q+4\left(\frac{4 \sqrt{6}}{5}-1\right)$, for $p, q>$ 1. Proof. By using the information about the edge partition given in Table 1, we have $G A(G)=\sum_{u v \in E(G)} 2 \frac{\sqrt{d_{u} d_{v}}}{\left(d_{u}+d_{v}\right)}=2 \frac{\sqrt{2 \times 3}}{2+3} \times 8+2 \frac{\sqrt{3 \times 3}}{3+3} \times$ $(6 p q+3 p+3 q-4)$.

$$
G A(G)=6 p q+3 p+3 q+4\left(\frac{4 \sqrt{6}}{5}-1\right) .
$$

Table 2. Edge partition of octo-nanowindow based on vertices degree sum which are located at unit distance from the final vertices of each edge.

\begin{tabular}{|l|l|}
\hline$\left(S_{u}, S_{v}\right)$ where $u v \in E(G)$ & Number of edges \\
\hline$(6,8)$ & 8 \\
\hline$(8,8)$ & 4 \\
\hline$(8,9)$ & 8 \\
\hline$(9,9)$ & $6 p q+3 p+3 q-16$ \\
\hline
\end{tabular}

In next two results, we shall calculate the $A B C_{4}$ and $G A_{5}$ index for the graph $G$ of octo-nanowindow.

Theorem 2.3 Let $G$ be the graph of octonanowindow. Then,

$$
A B C_{4}(G)=\left[\frac{8}{3}\right] p q+\frac{4}{3} p+\frac{4}{3} q-\frac{28}{9}+\frac{\sqrt{14}}{2}+\frac{2 \sqrt{30}}{3}
$$
holds for $p, q>1$.

Proof. The formula for $A B C_{4}$ index is

$$
A B C_{4}(G)=\sum_{u v \in E(G)} \sqrt{\frac{S_{u}+S_{v}-2}{S_{u} S_{v}}} .
$$

By using the information given in Table 2, we get

$$
\begin{gathered}
A B C_{4}(G)=8 \sqrt{\frac{6+8-2}{6 \times 8}}+4 \sqrt{\frac{8+8-2}{8 \times 8}}+ \\
8 \sqrt{\frac{8+9-2}{8 \times 9}}+(6 p q+3 p+3 q-16) \sqrt{\frac{9+9-2}{9 \times 9}}, \\
A B C_{4}(G)=\left[\frac{8}{3}\right] p q+\frac{4}{3} p+\frac{4}{3} q-\frac{28}{9}+\frac{\sqrt{14}}{2}+\frac{2 \sqrt{30}}{3} .
\end{gathered}
$$

Theorem 2.4 Suppose $G$ is the graph of octonanowindow. Then, $G A_{5}(G)=6 p q+3 p+3 q+$ $\frac{32 \sqrt{3}}{7}+\frac{96 \sqrt{3}}{17}-12$, holds for $p, q>1$.

Proof. By using the information in the second colmun of Table 2, we have $G A_{5}(G)=\sum_{u v \in E(G)} \frac{2 \sqrt{S_{u} S_{v}}}{\left(S_{u}+S_{v}\right)}$ $=\frac{2 \sqrt{6 \times 8}}{6+8} \times 8+\frac{2 \sqrt{8 \times 8}}{8+8} \times 4+\frac{2 \sqrt{8 \times 9}}{8+9} \times 8+\frac{2 \sqrt{9 \times 9}}{9+9} \times$ $(6 p q+3 p+3 q-16)$

This implies

$$
G A_{5}(G)=6 p q+3 p+3 q+\frac{32 \sqrt{3}}{7}+\frac{96 \sqrt{3}}{17}-12 .
$$




\section{Equilateral triangular tetra sheets network}

This Section is devoted to the study of degreebased topological descriptors of the equilateral triangular tetra sheet.

We denote a graph of equilateral triangular tetra sheet network with dimension $n$ by $\operatorname{ETTS}(n)$. The dimension $n$ of $\operatorname{ETTS}(n)$ is represented by arranging $n$ vertices on one side of the triangle structure.

\subsection{Construction of the graph $\operatorname{ETTS}(n)$}

First we draw a triangle having $n$ vertices on each side. We connect a vertex from one side of the triangle to the corresponding vertices of the other two sides of the triangle by an edge. Introduce new vertices in the intersecting edges. Replace all $K_{3}$ with $K_{4}$. (see Figure 3). The graph obtained in this way is called equilateral triangular tetra sheet with dimension $n$. The number of vertices and edges in $\operatorname{ETTS}(n)$ are $\frac{\left(3 n^{2}-3 n+2\right)}{2}$ and $\frac{\left(9 n^{2}-15 n+6\right)}{2}$ respectively.We note that the corner vertices of the graph $\operatorname{ETTS}(n)$ with degree $(3,3)$ are at equal distances to all other vertices for each $n$, where $n \geq 2$. In the following theorems, we compute the $A B C$ and $G A$ indices for the graph of equilateral triangular tetra sheet network.

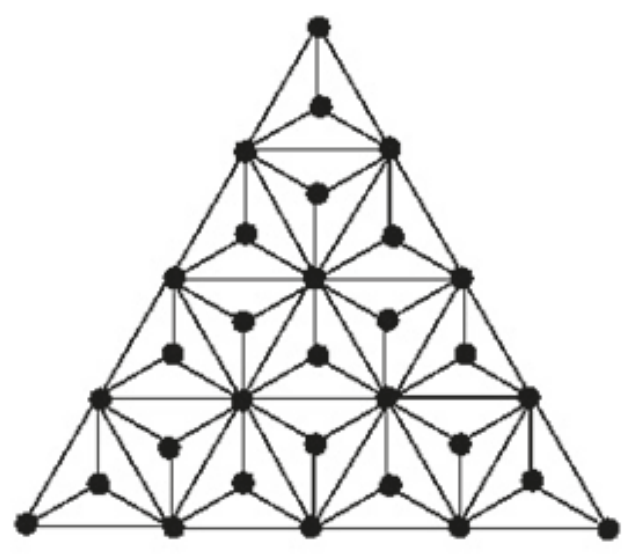

Figure 2. The equilateral tetra sheet network $\operatorname{ETTS}(5)$
Table 3. Edge partition of ETTS(5) based on degree of final vertices of each edge, where $u v \in E(G)$

\begin{tabular}{|c|c|}
\hline$\left(d_{u}, d_{v}\right)$ & Number of Edges \\
\hline$(3,3)$ & 3 \\
\hline$(3,7)$ & $9 n-12$ \\
\hline$(7,7)$ & $3 n-6$ \\
\hline$(3,12)$ & $3(n-3)(n-2)$ \\
\hline$(7,12)$ & $6 n-18$ \\
\hline$(12,12)$ & $\frac{3}{2}(n-4)(n-3)$ \\
\hline
\end{tabular}

Theorem 3.1 Suppose that Gis a graph such that $G \cong \operatorname{ETTS}(n)$. Then $\operatorname{ABC}(\operatorname{ETTS}(n))=$ $\left(n^{2}\right)\left[\frac{\sqrt{22}}{8}+\frac{\sqrt{13}}{2}\right]+n\left[\frac{6}{7}(\sqrt{42})+\frac{6}{7}(\sqrt{3})-\frac{5}{2}(\sqrt{13})+\right.$ $\left.\frac{\sqrt{357}}{7}-\frac{7}{8} \sqrt{22}\right]+\left[2+\frac{8}{7}(\sqrt{42})-\frac{12}{7}(\sqrt{3})+\right.$ $\left.3 \sqrt{13}-\frac{3}{7}(\sqrt{357})+\frac{3}{2}(\sqrt{22})\right]$, holds for $n \geq$ 3. Proof. By using the information about the edge partition given in Table 3, we have $A B C(G)=\sum_{u v \in E(G)} \sqrt{\frac{d_{u}+d_{v}-2}{d_{u} d_{v}}}=3 \sqrt{\frac{3+3-2}{3 \times 3}}+(9 n-$ 12) $\sqrt{\frac{3+7-2}{3 \times 7}}+(3 n-6) \sqrt{\frac{7+7-2}{7 \times 7}}+3(n-3)(n-$ 2) $\sqrt{\frac{3+12-2}{3 \times 12}}+(6 n-18) \sqrt{\frac{7+12-2}{7} \times 12}+\frac{3}{2}(n-$ 4) $(n-3) \sqrt{\frac{12+12-2}{12 \times 12}}$.

$$
A B C(G)=\left(n^{2}\right)\left[\frac{\sqrt{22}}{8}+\frac{\sqrt{13}}{2}\right]+n\left[\frac{6}{7}(\sqrt{42})\right.
$$
$\left.+\frac{6}{7}(\sqrt{3})-\frac{5}{2}(\sqrt{13})+\frac{\sqrt{357}}{7}-\frac{7}{8} \sqrt{22}\right]+[2+$ $\left.\frac{8}{7}(\sqrt{42})-\frac{12}{7}(\sqrt{3})+3 \sqrt{13}-\frac{3}{7}(\sqrt{357})+\frac{3}{2}(\sqrt{22})\right]$.

In the following theorem, we calculate the $G A$ index of $\operatorname{ETTS}(n)$

Theorem 3.2 Assume that $G$ is a graph of $\operatorname{ETTS}(n)$. Then, the following holds for $n \geq$ $3 G A(G)=n^{2}\left[\frac{39}{10}\right]+n\left[\frac{9}{5}(\sqrt{21})+\frac{24}{19}(\sqrt{21})-\frac{39}{2}\right]$ $+\left[\frac{147}{5}-12 \frac{\sqrt{21}}{5}+72 \frac{\sqrt{21}}{19}\right]$.

Proof. By following the information given in Table 3, we get $G A(G)=\sum_{u v \in E(G)} 2 \frac{\sqrt{d_{u} d_{v}}}{\left(d_{u}+d_{v}\right)}=3 \times$ $2 \frac{\sqrt{3 \times 3}}{3+3}+(9 n-12) \times 2 \frac{\sqrt{3 \times 7}}{3+7}+(3 n-6) \times 2 \frac{\sqrt{7 \times 7}}{7+7}$ $+3(n-3)(n-2) \times 2 \frac{\sqrt{3 \times 12}}{3+12}+(6 n-18) \times 2 \frac{\sqrt{7 \times 12}}{7+12}$ $+\frac{3}{2}(n-4)(n-3) \times 2 \frac{\sqrt{12 \times 12}}{12+12}$.

$G A(G)=n^{2}\left[\frac{39}{10}\right]+n\left[\frac{9}{5}(\sqrt{21})+\frac{24}{19}(\sqrt{21})-\frac{39}{2}\right]$ $+\left[\frac{147}{5}-12 \frac{\sqrt{21}}{5}+72 \frac{\sqrt{21}}{19}\right]$. 
Table 4. Edge Partition of ETTS $(n)$ based on vertices degree sum which are located at unit distance from the final vertices of each edge.

\begin{tabular}{|c|c|}
\hline$\left(S_{u}, S_{v}\right)$ whereuv $\in E(G)$ & Number of edges \\
\hline$(17,17)$ & 3 \\
\hline$(17,38)$ & 12 \\
\hline$(38,38)$ & 3 \\
\hline$(38,47)$ & 6 \\
\hline$(38,70)$ & 6 \\
\hline$(47,70)$ & 6 \\
\hline$(70,80)$ & 6 \\
\hline$(38,26)$ & 12 \\
\hline$(26,70)$ & 9 \\
\hline$(31,70)$ & 6 \\
\hline$(36,70)$ & 3 \\
\hline$(47,80)$ & $6(n-5)$ \\
\hline$(80,80)$ & $3(n-5)$ \\
\hline$(47,47)$ & $3(n-5)$ \\
\hline$(80,90)$ & $6(n-6)$ \\
\hline$(90,90)$ & $3\left(n^{2}-13 n+42\right)$ \\
\hline$(47,26)$ & $6(n-4)$ \\
\hline$(26,80)$ & $3(n-5)$ \\
\hline$(47,31)$ & $3(n-4)$ \\
\hline$(31,80)$ & $6(n-5)$ \\
\hline$(36,80)$ & $9(n-5)$ \\
\hline$(36,90)$ & $3\left(n^{2}-11 n+30\right)$ \\
\hline
\end{tabular}

In Theorem 3.3 and Theorem 3.4, we are in the position to compute the $A B C_{4}$ and $G A_{5}$ indices of a graph $G$, where $G \cong \operatorname{ETTS}(n)$. For this purpose, the edge partition of $G$ based on the degree sum of final vertices of each edge is given in the following Table.

Theorem 3.3 For $n \geq 6$, the $A B C_{4}$ index of a graph $G \cong \operatorname{ETTS}(n)$ is $\left.A B C_{4}(G)\right)=\left(\frac{\sqrt{89}}{30 \sqrt{2}}+\frac{\sqrt{31}}{3 \sqrt{10}}\right) n^{2}+$ $\left(\frac{15}{2 \sqrt{47}}+\frac{3 \sqrt{79}}{40 \sqrt{2}}+6 \sqrt{\frac{23}{2209}}+\frac{3 \sqrt{7}}{5 \sqrt{3}}-\frac{13 \sqrt{89}}{30 \sqrt{2}}+6 \sqrt{\frac{71}{1222}}+\right.$ $\left.\frac{3}{2 \sqrt{5}}+6 \sqrt{\frac{19}{1457}}+\frac{3 \sqrt{109}}{2 \sqrt{155}}+\frac{9 \sqrt{19}}{4 \sqrt{30}}-\frac{11 \sqrt{31}}{3 \sqrt{10}}\right) n+$ $\frac{12 \sqrt{2}}{17}+12 \sqrt{\frac{53}{646}}+\frac{3 \sqrt{37}}{19 \sqrt{2}}+6 \sqrt{\frac{83}{1786}}+6 \sqrt{\frac{53}{1330}}+$ $6 \sqrt{\frac{23}{658}}+\frac{3 \sqrt{37}}{5 \sqrt{14}}+12 \sqrt{\frac{31}{494}}+9 \sqrt{\frac{47}{910}}+18 \sqrt{\frac{11}{2170}}+$ $\sqrt{\frac{13}{35}}-\frac{75}{2 \sqrt{47}}-\frac{3 \sqrt{79}}{8 \sqrt{2}}-30 \sqrt{\frac{23}{2209}}-\frac{18 \sqrt{7}}{5 \sqrt{3}}+\frac{7 \sqrt{89}}{5 \sqrt{2}}-$ $24 \sqrt{\frac{71}{1222}}-\frac{15}{2 \sqrt{5}}-24 \sqrt{\frac{19}{1457}}-\frac{15 \sqrt{109}}{2 \sqrt{155}}-\frac{45 \sqrt{19}}{4 \sqrt{30}}+$ $10 \sqrt{\frac{31}{10}}$.

Proof. Since we know that, the formula for computing $A B C_{4}$ index of the given graph is
$A B C_{4}(G)=\sum_{u v \in E(G)} \sqrt{\frac{S_{u}+S_{v}-2}{S_{u} S_{v}}}$. By inserting the information given in Table 4, whave $A B C_{4}(G)=$ $3 \sqrt{\frac{17+17-2}{17 \times 17}}+12 \sqrt{\frac{17+38-2}{17 \times 38}}+3 \sqrt{\frac{38+38-2}{38 \times 38}}+$ $6 \sqrt{\frac{38+47-2}{38 \times 47}}+6 \sqrt{\frac{38+70-2}{38 \times 70}}+6 \sqrt{\frac{47+70-2}{47 \times 70}}+$ $6 \sqrt{\frac{70+80-2}{70 \times 80}}+12 \sqrt{\frac{38+26-2}{38 \times 26}}+9 \sqrt{\frac{26+70-2}{26 \times 70}}+$ $6 \sqrt{\frac{31+70-2}{31 \times 70}}+3 \sqrt{\frac{36+70-2}{36 \times 70}}+6(n-5) \sqrt{\frac{47+80-2}{47 \times 80}}+$ $3(n-5) \sqrt{\frac{80+80-2}{80 \times 80}}+3(n-5) \sqrt{\frac{47+47-2}{47 \times 47}}+$ $6(n-6) \sqrt{\frac{80+90-2}{80 \times 90}}+\left(\frac{3\left(n^{2}-13 n+42\right)}{2}\right) \sqrt{\frac{90+90-2}{90 \times 90}}+$ $6(n-4) \sqrt{\frac{47+26-2}{47 \times 26}}+3(n-5) \sqrt{\frac{26+80-2}{26 \times 80}}+$ $3(n-4) \sqrt{\frac{47+31-2}{47 \times 31}}+6(n-5) \sqrt{\frac{31+80-2}{31 \times 80}}+9(n-$ 5) $\sqrt{\frac{36+80-2}{36 \times 80}}+3\left(n^{2}-11 n+30\right) \sqrt{\frac{36+90-2}{36 \times 90}}$

$A B C_{4}(G)=3 \sqrt{\frac{17+17-2}{17 \times 17}}+12 \sqrt{\frac{17+38-2}{17 \times 38}}+$
$3 \sqrt{\frac{38+38-2}{38 \times 38}}+6 \sqrt{\frac{38+47-2}{38 \times 47}}+6 \sqrt{\frac{38+70-2}{38 \times 70}}+$
$6 \sqrt{\frac{47+70-2}{47 \times 70}}+6 \sqrt{\frac{70+80-2}{70 \times 80}}+12 \sqrt{\frac{38+26-2}{38 \times 26}}+$
$9 \sqrt{\frac{26+70-2}{26 \times 70}}+6 \sqrt{\frac{31+70-2}{31 \times 70}}+3 \sqrt{\frac{36+70-2}{36 \times 70}}+$
$6(n-5) \sqrt{\frac{47+80-2}{47 \times 80}}+3(n-5) \sqrt{\frac{80+80-2}{80 \times 80}}+$
$3(n-5) \sqrt{\frac{47+47-2}{47 \times 47}}+6(n-6) \sqrt{\frac{80+90-2}{80 \times 90}}+$
$\left(\frac{3\left(n^{2}-13 n+42\right)}{2}\right) \sqrt{\frac{90+90-2}{90 \times 90}}+6(n-4) \sqrt{\frac{47+26-2}{47 \times 26}}+$ $3(n-5) \sqrt{\frac{26+80-2}{26 \times 80}}+3(n-4) \sqrt{\frac{47+31-2}{47 \times 31}}+6(n-$ 5) $\sqrt{\frac{31+80-2}{31 \times 80}}+9(n-5) \sqrt{\frac{36+80-2}{36 \times 80}}+3\left(n^{2}-\right.$ $11 n+30) \sqrt{\frac{36+90-2}{36 \times 90}} \quad A B C_{4}(G)=3 \sqrt{\frac{17+17-2}{17 \times 17}}+$ $12 \sqrt{\frac{17+38-2}{17 \times 38}}+3 \sqrt{\frac{38+38-2}{38 \times 38}}+6 \sqrt{\frac{38+47-2}{38 \times 47}}+$ $6 \sqrt{\frac{38+70-2}{38 \times 70}}+6 \sqrt{\frac{47+70-2}{47 \times 70}}+6 \sqrt{\frac{70+80-2}{70 \times 80}}+$ $12 \sqrt{\frac{38+26-2}{38 \times 26}}+9 \sqrt{\frac{26+70-2}{26 \times 70}}+6 \sqrt{\frac{31+70-2}{31 \times 70}}+$ $3 \sqrt{\frac{36+70-2}{36 \times 70}}+6(n-5) \sqrt{\frac{47+80-2}{47 \times 80}}+3(n-$ 5) $\sqrt{\frac{80+80-2}{80 \times 80}}+3(n-5) \sqrt{\frac{47+47-2}{47 \times 47}}+6(n-$ 6) $\sqrt{\frac{80+90-2}{80 \times 90}}+\left(\frac{3\left(n^{2}-13 n+42\right)}{2}\right) \sqrt{\frac{90+90-2}{90 \times 90}}+6(n-$ 4) $\sqrt{\frac{47+26-2}{47 \times 26}}+3(n-5) \sqrt{\frac{26+80-2}{26 \times 80}}+3(n-$ 4) $\sqrt{\frac{47+31-2}{47 \times 31}}+6(n-5) \sqrt{\frac{31+80-2}{31 \times 80}}+9(n-$ 5) $\sqrt{\frac{36+80-2}{36 \times 80}}+3\left(n^{2}-11 n+30\right) \sqrt{\frac{36+90-2}{36 \times 90}}$

Further simplification gives us the following form $\left.A B C_{4}(G)=\frac{\sqrt{89}}{30 \sqrt{2}}+\frac{\sqrt{31}}{3 \sqrt{10}}\right) n^{2}+\left(\frac{15}{2 \sqrt{47}}+\right.$ $\frac{3 \sqrt{79}}{40 \sqrt{2}}+6 \sqrt{\frac{23}{2209}}+\frac{3 \sqrt{7}}{5 \sqrt{3}}-\frac{13 \sqrt{89}}{30 \sqrt{2}}+6 \sqrt{\frac{71}{1222}}+$ 


$$
\begin{aligned}
& \left.\frac{3}{2 \sqrt{5}}+6 \sqrt{\frac{19}{1457}}+\frac{3 \sqrt{109}}{2 \sqrt{155}}+\frac{9 \sqrt{19}}{4 \sqrt{30}}-\frac{11 \sqrt{31}}{3 \sqrt{10}}\right) n+ \\
& \left(\frac{12 \sqrt{2}}{17}+12 \sqrt{\frac{53}{646}}+\frac{3 \sqrt{37}}{19 \sqrt{2}}+6 \sqrt{\frac{83}{1786}}+6 \sqrt{\frac{53}{1330}}+\right. \\
& 6 \sqrt{\frac{23}{658}}+\frac{3 \sqrt{37}}{5 \sqrt{14}}+12 \sqrt{\frac{31}{494}}+9 \sqrt{\frac{47}{910}}+18 \sqrt{\frac{11}{2170}}+ \\
& \sqrt{\frac{13}{35}}-\frac{75}{2 \sqrt{47}}-\frac{3 \sqrt{79}}{8 \sqrt{2}}-30 \sqrt{\frac{23}{2209}}-\frac{18 \sqrt{7}}{5 \sqrt{3}}+\frac{7 \sqrt{89}}{5 \sqrt{2}}- \\
& 24 \sqrt{\frac{71}{1222}}-\frac{15}{2 \sqrt{5}}-24 \sqrt{\frac{19}{1457}}-\frac{15 \sqrt{109}}{2 \sqrt{155}}-\frac{45 \sqrt{19}}{4 \sqrt{30}}+ \\
& 10 \sqrt{\frac{31}{10}} .
\end{aligned}
$$

Theorem 3.4 For $n \geq 6$, the GA5 index of a graph $G$ of the equilateral triangular tetra sheet $\operatorname{ETTS}(n)$ is $G A_{5}(G)=\left(\frac{6 \sqrt{10}}{7}+\frac{3}{2}\right) n^{2}+\left(\frac{48 \sqrt{235}}{127}-\right.$ $\frac{27}{2}+\frac{72 \sqrt{2}}{17}+\frac{12 \sqrt{1222}}{73}+\frac{12 \sqrt{130}}{53}+\frac{\sqrt{1457}}{13}+\frac{48 \sqrt{155}}{111}+$ $\left.\frac{108 \sqrt{5}}{29}-\frac{66 \sqrt{10}}{7}\right) n+\left(\frac{24 \sqrt{646}}{55}+\frac{12 \sqrt{1786}}{85}+\frac{\sqrt{2660}}{9}+\right.$ $\frac{4 \sqrt{3290}}{39}+\frac{8 \sqrt{14}}{5}+\frac{3 \sqrt{247}}{4}+\frac{3 \sqrt{455}}{8}+\frac{12 \sqrt{2170}}{101}+\frac{18 \sqrt{70}}{53}-$ $\frac{240 \sqrt{235}}{127}-\frac{432 \sqrt{2}}{17}-\frac{48 \sqrt{1222}}{73}-\frac{60 \sqrt{130}}{53}-\frac{4 \sqrt{1457}}{13}-$ $\frac{240 \sqrt{155}}{111}-\frac{540 \sqrt{5}}{29}+\frac{180 \sqrt{10}}{7}+39$ ).

Proof. Inserting the information about the edge partitition from Table 4, in the following, $A_{5}(G)=$ $\sum_{u v \in E(G)} 2 \frac{\sqrt{S_{u} S_{v}}}{S_{u}+S_{v}}=3 \times \frac{2 \sqrt{17 \times 17}}{17+17}+12 \times \frac{2 \sqrt{17 \times 38}}{17+38}+$ $3 \times \frac{2 \sqrt{38 \times 38}}{38+38}+6 \times \frac{2 \sqrt{38 \times 47}}{38+47}+6 \times \frac{2 \sqrt{38 \times 70}}{38+70}+6 \times$ $\frac{2 \sqrt{47 \times 70}}{47+70}+6 \times \frac{2 \sqrt{70 \times 80}}{70+80}+12 \times \frac{2 \sqrt{38 \times 26}}{38+26}+9 \times$ $\frac{2 \sqrt{26 \times 70}}{26+70}+6 \times \frac{2 \sqrt{31 \times 70}}{31+70}+3 \times \frac{2 \sqrt{36 \times 70}}{36+70}+6(n-$ 5) $\times \frac{2 \sqrt{47 \times 80}}{47+80}+3(n-5) \times \frac{2 \sqrt{80 \times 80}}{80+80}+3(n-5) \times$ $\frac{2 \sqrt{47 \times 47}}{47+47}+6(n-6) \times \frac{2 \sqrt{80 \times 90}}{80+90}+\frac{3\left(n^{2}-13 n+42\right)}{2} \times$ $\frac{2 \sqrt{90 \times 90}}{90+90}+6(n-4) \times \frac{2 \sqrt{47 \times 26}}{47+26}+3(n-5) \times$ $\frac{2 \sqrt{26 \times 80}}{26+80}+3(n-4) \times \frac{2 \sqrt{47 \times 31}}{47+31}+6(n-5) \times$ $\frac{2 \sqrt{31 \times 80}}{31+80}+9(n-5) \times \frac{2 \sqrt{36 \times 80}}{36+80}+3\left(n^{2}-11 n+30\right) \times$ $\frac{2 \sqrt{36 \times 90}}{36+90}$.

After simplification, we get $G A_{5}(G)=\left(\frac{6 \sqrt{10}}{7}+\right.$ $\left.\frac{3}{2}\right) n^{2}+\left(\frac{48 \sqrt{235}}{127}-\frac{27}{2}+\frac{72 \sqrt{2}}{17}+\frac{12 \sqrt{1222}}{73}+\frac{12 \sqrt{130}}{53}+\right.$ $\left.\frac{\sqrt{1457}}{13}+\frac{48 \sqrt{155}}{111}+\frac{108 \sqrt{5}}{29}-\frac{66 \sqrt{10}}{7}\right) n+\left(\frac{24 \sqrt{646}}{55}+\right.$ $\frac{12 \sqrt{1786}}{85}+\frac{\sqrt{2660}}{9}+\frac{4 \sqrt{3290}}{39}+\frac{8 \sqrt{14}}{5}+\frac{3 \sqrt{247}}{4}+\frac{3 \sqrt{455}}{8}+$ $\frac{12 \sqrt{2170}}{101}+\frac{18 \sqrt{70}}{53}-\frac{240 \sqrt{235}}{127}-\frac{432 \sqrt{2}}{17}-\frac{48 \sqrt{1222}}{73}-$ $\left.\frac{60 \sqrt{130}}{53}-\frac{4 \sqrt{1457}}{13}-\frac{240 \sqrt{155}}{111}-\frac{540 \sqrt{5}}{29}+\frac{180 \sqrt{10}}{7}+39\right)$.

\section{Extended mesh or rectangular network sheet}

This Section is about the study of degree-based topological descriptors for the extended mesh.

\subsection{Construction of extended mesh net- work}

Assume that $P_{n}$ is a path with $n$ vertices. Th $P_{m} \times P_{n}$, form, $n \geq 2$, represents the two dimensional mesh, where $m$ is the number of rows and $n$ is the number of columns. We denote this by $M(m, n)$. Note that $M(m, n)$ is a graph with vertex set $V(M(m, n))=v_{i j}, 1 \leq i \leq m, 1 \leq j \leq n$ and edge set $E(M(m, n))=\left(v_{i j}, v_{i(j+1)}\right): 1 \leq i \leq m, 1 \leq j \leq$ $n-1 \cup v_{i j}, v_{(i+1) j}: 1 \leq i \leq m-1,1 \leq j \leq n$.

By constructing all 4-cycle in a $m \times n$ mesh into a complete subgraph, we get an architecture called the extended mesh that is denoted by $E X(m, n)$. The number of vertices in $E X(m, n)$ is $m n$ and the number of edges in $E X(m, n)$ is $4 m n-3 m-3 n+2$.

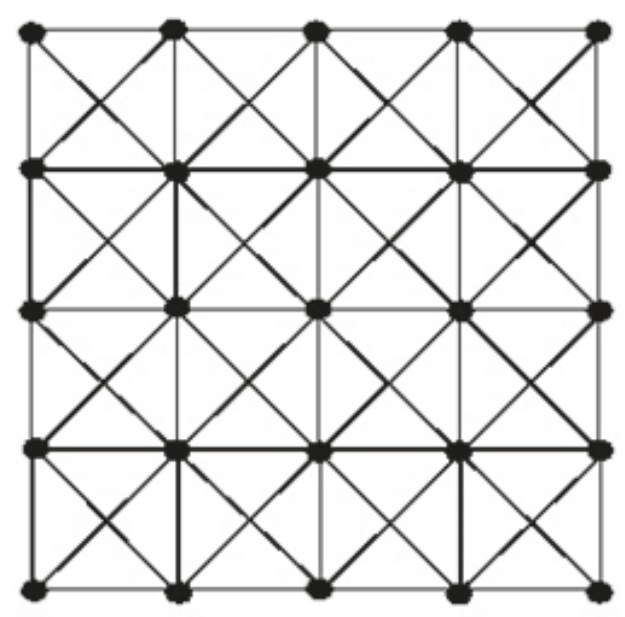

Figure 3. The rectangular sheet network (extended mesh) $E X(5,5)$

Table 5. Edge partition of $E X(m, n)$, based on degree of end vertices of each edge, where $u v \in E(G)$.

\begin{tabular}{|c|c|}
\hline$\left(d_{u}, d_{v}\right)$ & Number of Edges \\
\hline$(3,5)$ & 8 \\
\hline$(3,8)$ & 4 \\
\hline$(5,8)$ & $(6 m+6 n-32)$ \\
\hline$(5,5)$ & $2(m+n-4)$ \\
\hline$(8,8)$ & $(4 m n-11 m-11 n+30)$ \\
\hline
\end{tabular}


Theorem 4.1 The ABC index of extended mesh $E X(m, n)$ is

$$
A B C(E X(m, n))=\left(\sqrt{\frac{7}{2}}\right) m n+\left(3 \sqrt{\frac{11}{10}}+\frac{4 \sqrt{2}}{5}-\right.
$$

$\left.\frac{11 \sqrt{7}}{4 \sqrt{2}}\right) m+\left(3 \sqrt{\frac{11}{10}}+\frac{4 \sqrt{2}}{5}-\frac{11 \sqrt{7}}{4 \sqrt{2}}\right) n+\left(8 \sqrt{\frac{2}{5}}+2 \sqrt{\frac{3}{2}}-\right.$ $\left.16 \sqrt{\frac{11}{10}}-\frac{16 \sqrt{2}}{5}+\frac{15 \sqrt{7}}{2 \sqrt{2}}\right)$, for $m, n \geq 5$.

Proof. Let $G$ be a graph of extended mesh $E X(m, n)$. The information given in Table 5 leads us to the following calculation

$$
A B C(G)=\sum_{u v \in E(G)} \sqrt{\frac{d_{u}+d_{v}-2}{d_{u} d_{v}}}=8 \sqrt{\frac{3+5-2}{3 \times 5}}+
$$

$4 \sqrt{\frac{3+8-2}{3 \times 8}}+(6 m+6 n-32) \sqrt{\frac{5+8-2}{5 \times 8}}+2(m+n-$ 4) $\sqrt{\frac{5+5-2}{5 \times 5}}+(4 m n-11 m-11 n+30) \sqrt{\frac{8+8-2}{8 \times 8}}$.

More simplification reduce the above exprssion in to the following form $A B C(E X(m, n))=$ $\left(\sqrt{\frac{7}{2}}\right) m n+\left(3 \sqrt{\frac{11}{10}}+\frac{4 \sqrt{2}}{5}-\frac{11 \sqrt{7}}{4 \sqrt{2}}\right) m+\left(3 \sqrt{\frac{11}{10}}+\right.$ $\left.\frac{4 \sqrt{2}}{5}-\frac{11 \sqrt{7}}{4 \sqrt{2}}\right) n+\left(8 \sqrt{\frac{2}{5}}+2 \sqrt{\frac{3}{2}}-16 \sqrt{\frac{11}{10}}-\frac{16 \sqrt{2}}{5}+\right.$ $\left.\frac{15 \sqrt{7}}{2 \sqrt{2}}\right)$.

Table 6. Edge Partition of $E X(m, n)$ based on vertices degree sum which are located at unit distance from the final vertices of each edge.

\begin{tabular}{|c|c|}
\hline $\begin{array}{c}\left(S_{u}, S_{v}\right) \text { where } \\
u v \in E(G)\end{array}$ & Number of edges \\
\hline$(18,29)$ & 8 \\
\hline$(29,34)$ & 8 \\
\hline$(18,47)$ & 4 \\
\hline$(29,55)$ & 8 \\
\hline$(29,29)$ & 4 \\
\hline$(47,64)$ & 4 \\
\hline$(47,55)$ & 8 \\
\hline$(34,47)$ & 8 \\
\hline$(29,47)$ & 8 \\
\hline$(34,34)$ & $2(m+n-10)$ \\
\hline$(55,55)$ & $2(m+n-8)$ \\
\hline$(55,64)$ & $(6 m-6 n-56)$ \\
\hline$(34,55)$ & $(6 m+6 n-56)$ \\
\hline$(64,64)$ & $(4 m n-19 m-19 n+90)$ \\
\hline
\end{tabular}

Theorem 4.2 For $m, n \geq 5$, $G A(E X(m, n))=4 m n+$ $\left(\frac{24 \sqrt{10}}{13}-9\right) m+\left(\frac{24 \sqrt{10}}{13}-9\right) n+\left(2 \sqrt{15}+\frac{16 \sqrt{6}}{11}-\right.$ $\left.\frac{128 \sqrt{10}}{13}+22\right)$.

Proof. To compute the $G A$ index of the graph $G=E X(m, n)$, use the edge partition given in Table 5 to get $G A(G)=\sum_{u v \in E(G)} 2 \frac{\sqrt{d_{u} d_{v}}}{\left(d_{u}+d_{v}\right)}=8 \times \frac{2 \sqrt{3 \times 5}}{3+5}+$
$4 \times \frac{2 \sqrt{3 \times 8}}{3+8}+(6 m+6 n-32) \times \frac{2 \sqrt{5 \times 8}}{5+8}+2(m+n-$ 4) $\times \frac{2 \sqrt{5 \times 5}}{5+5}+(4 m n-11 m-11 n+30) \frac{2 \sqrt{8 \times 8}}{8+8}$

simple calculation implies $G A(E X(m, n))=$ $4 m n+\left(\frac{24 \sqrt{10}}{13}-9\right) m+\left(\frac{24 \sqrt{10}}{13}-9\right) n+(2 \sqrt{15}+$ $\left.\frac{16 \sqrt{6}}{11}-\frac{128 \sqrt{10}}{13}+22\right)$ The next two results are about the computation of the $A B C_{4}$ and $G A_{5}$ indices of the graph of $E X(m, n)$. We give the edge partition of a graph $G$, where $G \cong E X(m, n)$, based on the degree sum of the final vertices of each edge in the following table.

Theorem 4.3 For $m, n \geq 5$, the $A B C_{4}$ index of $E X(m, n)$ is computed as

$A B C_{4}(E X(m, n))=\left(\frac{3 \sqrt{7}}{8 \sqrt{2}}\right) m n+\left(\frac{2 \sqrt{33}}{17 \sqrt{2}}+\frac{12 \sqrt{3}}{55}+\right.$ $\left.\frac{9 \sqrt{13}}{4 \sqrt{55}}+6 \sqrt{\frac{87}{1870}}-\frac{57 \sqrt{7}}{32 \sqrt{2}}\right) m+\left(\frac{2 \sqrt{33}}{17 \sqrt{2}}+\frac{12 \sqrt{3}}{55}+\frac{9 \sqrt{13}}{4 \sqrt{55}}+\right.$ $\left.6 \sqrt{\frac{87}{1870}}-\frac{57 \sqrt{7}}{32 \sqrt{2}}\right) n+\left(8 \sqrt{\frac{5}{58}}+8 \sqrt{\frac{61}{986}}+4 \sqrt{\frac{7}{94}}+\right.$ $8 \sqrt{\frac{82}{1595}}+8 \sqrt{\frac{14}{841}}+\frac{\sqrt{109}}{2 \sqrt{47}}+16 \sqrt{\frac{5}{517}}+8 \sqrt{\frac{79}{1598}}+$ $8 \sqrt{\frac{74}{1363}}-\frac{20 \sqrt{33}}{17 \sqrt{2}}-\frac{96 \sqrt{3}}{55}-21 \sqrt{\frac{13}{55}}-56 \sqrt{\frac{87}{1870}}+$ $\left.\frac{135 \sqrt{7}}{16 \sqrt{2}}\right)$.

Proof. By following the data in Table 6, we have

$A B C_{4}(G)=\sum_{u v \in E(G)} \sqrt{\frac{S_{u}+S_{v}-2}{S_{u} S_{v}}}=8 \sqrt{\frac{18+29-2}{18 \times 29}}+$ $8 \sqrt{\frac{29+34-2}{29 \times 34}}+4 \sqrt{\frac{18+47-2}{18 \times 47}}+8 \sqrt{\frac{29+55-2}{29 \times 55}}+$ $4 \sqrt{\frac{29+29-2}{29 \times 29}}+4 \sqrt{\frac{47+64-2}{47 \times 64}}+8 \sqrt{\frac{47+55-2}{47 \times 55}}+$ $8 \sqrt{\frac{34+47-2}{34 \times 47}}+8 \sqrt{\frac{29+47-2}{29 \times 47}}+2(m+n-10) \sqrt{\frac{34+34-2}{34 \times 34}}+$ $2(m+n-8) \sqrt{\frac{55+55-2}{55 \times 55}}+(6 m+6 n-56) \sqrt{\frac{55+64-2}{55 \times 64}}+$ $(6 m+6 n-56) \sqrt{\frac{34+55-2}{34 \times 55}}+(4 m n-19 m-19 n+$ 90) $\sqrt{\frac{64+64-2}{64 \times 64}}$.

$A B C_{4}(E X(m, n))=\left(\frac{3 \sqrt{7}}{8 \sqrt{2}}\right) m n+\left(\frac{2 \sqrt{33}}{17 \sqrt{2}}+\frac{12 \sqrt{3}}{55}+\right.$ $\left.\frac{9 \sqrt{13}}{4 \sqrt{55}}+6 \sqrt{\frac{87}{1870}}-\frac{57 \sqrt{7}}{32 \sqrt{2}}\right) m+\left(\frac{2 \sqrt{33}}{17 \sqrt{2}}+\frac{12 \sqrt{3}}{55}+\frac{9 \sqrt{13}}{4 \sqrt{55}}+\right.$ $\left.6 \sqrt{\frac{87}{1870}}-\frac{57 \sqrt{7}}{32 \sqrt{2}}\right) n+\left(8 \sqrt{\frac{5}{58}}+8 \sqrt{\frac{61}{986}}+4 \sqrt{\frac{7}{94}}+\right.$ $8 \sqrt{\frac{82}{1595}}+8 \sqrt{\frac{14}{841}}+\frac{\sqrt{109}}{2 \sqrt{47}}+16 \sqrt{\frac{5}{517}}+8 \sqrt{\frac{79}{1598}}+$ $8 \sqrt{\frac{74}{1363}}-\frac{20 \sqrt{33}}{17 \sqrt{2}}-\frac{96 \sqrt{3}}{55}-21 \sqrt{\frac{13}{55}}-56 \sqrt{\frac{87}{1870}}+$ $\left.\frac{135 \sqrt{7}}{16 \sqrt{2}}\right)$.

Theorem 4.4 For $m, n \geq 5$, we have $G A_{5}(E X(m, n))=4 m n+\left(\frac{12 \sqrt{1870}}{89}-\frac{1689}{119}\right) m+$ $\left(\frac{12 \sqrt{1870}}{89}-\frac{1689}{119}\right) n+\left(\frac{48 \sqrt{58}}{47}+\frac{16 \sqrt{986}}{63}+\frac{24 \sqrt{94}}{65}+\right.$ $\frac{4 \sqrt{1595}}{21}+\frac{4 \sqrt{841}}{29}+\frac{64 \sqrt{47}}{111}+\frac{8 \sqrt{2585}}{51}+\frac{16 \sqrt{1598}}{81}-$ 
$\left.\frac{4 \sqrt{1363}}{19}-\frac{112 \sqrt{1870}}{89}+\frac{5530}{119}\right)$.

Proof. The information in Table 6 leads us to the required result.

$$
G A_{5}(G)=\sum_{u v \in E(G)} 2 \frac{\sqrt{S_{u} S_{v}}}{S_{u}+S_{v}}=8 \times \frac{2 \sqrt{18 \times 29}}{18+29}+
$$

$8 \times \frac{2 \sqrt{29 \times 34}}{29+34}+4 \times \frac{2 \sqrt{18 \times 47}}{18+47}+8 \times \frac{2 \sqrt{29 \times 55}}{29+55}+4 \times$ $\frac{2 \sqrt{29 \times 29}}{29+29}+4 \times \frac{2 \sqrt{47 \times 64}}{47+64}+8 \times \frac{2 \sqrt{47 \times 55}}{47+55}+8 \times$ $\frac{2 \sqrt{34 \times 47}}{34+47}+8 \times \frac{2 \sqrt{29 \times 47}}{29+47}+2(m+n-10) \times \frac{2 \sqrt{34 \times 34}}{34+34}+$ $2(m+n-8) \times \frac{2 \sqrt{55 \times 55}}{55+55}+(6 m+6 n-56) \times$ $\frac{2 \sqrt{55 \times 64}}{55+64}+(6 m+6 n-56) \times \frac{2 \sqrt{34 \times 55}}{34+55}+(4 m n-$ $19 m-19 n+90) \times \frac{2 \sqrt{64 \times 64}}{64+64}$.

After simplification,we get

$G A_{5}(E X(m, n))=4 m n+\left(\frac{12 \sqrt{1870}}{89}-\frac{1689}{119}\right) m+$ $\left(\frac{12 \sqrt{1870}}{89}-\frac{1689}{119}\right) n+\left(\frac{48 \sqrt{58}}{47}+\frac{16 \sqrt{986}}{63}+\frac{24 \sqrt{94}}{65}+\right.$ $\frac{4 \sqrt{1595}}{21}+\frac{4 \sqrt{841}}{29}+\frac{64 \sqrt{47}}{111}+\frac{8 \sqrt{2585}}{51}+\frac{16 \sqrt{1598}}{81}-$ $\left.\frac{4 \sqrt{1363}}{19}-\frac{112 \sqrt{1870}}{89}+\frac{5530}{119}\right)$.

\section{Rectangular tetra sheets network}

In this Section, we compute the $A B C, A B C_{4}$, $G A$, and $G A_{5}$ indices of a graph of rectangular tetra sheet network.

\subsection{Construction of Rectangular Tetra Sheet}

Draw a grid graph $P_{m} P_{n}$ of dimension $(m, n)$. Join the diagonal vertices of the paraellelogram so that each rectangle is divided into two triangles. Replace each triangle $K_{3}$ by $K_{4}$, the resulting graph is known as the graph of rectangular tetra sheet network and is denoted by $\operatorname{RTS}(m, n)$, (see Figure 4). In this graph, the number of vertices and edges are $3 m n-2(m+n)+2$ and $9 m n-8 n-8 m+7$ respectively.

In the next two theorems, we compute the $A B C$ and $G A$ indices of the graph of rectangular tetra sheet network by using the edge partition given in the following table.
Table 7. Edge partition of $R T S(m, n)$. It depends on the degrees of the final vertices located at unit distance of each edge.

\begin{tabular}{|c|c|}
\hline $\begin{array}{c}\left(d_{u}, d_{v}\right) \text { where } \\
u v \in E(G)\end{array}$ & Number of Edges \\
\hline$(3,3)$ & 2 \\
\hline$(5,7)$ & 4 \\
\hline$(5,12)$ & 2 \\
\hline$(3,5)$ & 4 \\
\hline$(3,7)$ & $6 m+6 n-20$ \\
\hline$(3,12)$ & $6 m n-12 m-12 n+24$ \\
\hline$(7,7)$ & $2 m+2 n-10$ \\
\hline$(7,12)$ & $4 m+4 n-20$ \\
\hline$(12,12)$ & $3 m n-8 m-8 n+21$ \\
\hline
\end{tabular}

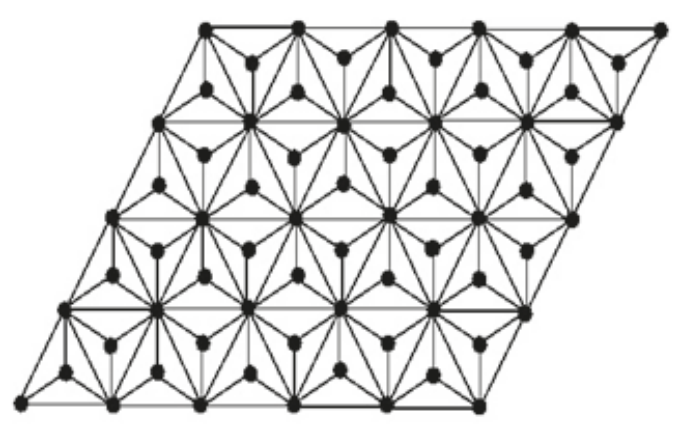

Figure 4. The graph of rectangular tetra sheet $\operatorname{RTS}(m, n)$ where $m=5$ and $n=6$

Theorem 5.1 Let $G \cong R T S(m, n)$. Then, for $m, n \geq$ 3, the $A B C(G)$ is

$A B C(G)=\left(\sqrt{13}+\frac{\sqrt{22}}{4}\right) m n+\left[\frac{4}{7} \sqrt{3}+\frac{4}{7} \sqrt{42}-\right.$ $\left.\frac{2}{3} \sqrt{22}-2 \sqrt{13}+\frac{2}{21} \sqrt{357}\right](m+n) \frac{4}{3}+\frac{4}{7} \sqrt{14}+$ $\frac{4}{5} \sqrt{10}+4 \sqrt{13}-\frac{10}{21} \sqrt{357}-\frac{20}{7} \sqrt{3}-\frac{40}{21} \sqrt{42}+$ $\frac{7}{4} \sqrt{22}+1$.

Proof. Put the information given in Table 7 in $A B C(G)=\sum_{u v \in E(G)} \sqrt{\frac{d_{u}+d_{v}-2}{d_{u} d_{v}}}$, we have $A B C(G)=2 \sqrt{\frac{3+3-2}{3 \times 3}}+4 \sqrt{\frac{5+7-2}{5 \times 7}}+2 \sqrt{\frac{5+12-2}{5 \times 12}}+$ $4 \sqrt{\frac{3+5-2}{3 \times 5}}+(6(m-2)(n-2)) \sqrt{\frac{3+12-2}{3 \times 12}}+(4 m+$ $4 n-20) \sqrt{\frac{7+12-2}{7 \times 12}}+(2 m+2 n-10) \sqrt{\frac{7+17-2}{7 \times 7}}+$ $(6 m+6 n-20) \sqrt{\frac{3+7-2}{3 \times 17}}+(3 m n-8 m-8 n+$ 21) $\sqrt{\frac{12+12-2}{12 \times 12}}$

Further simplification reduces the above expression to the following form

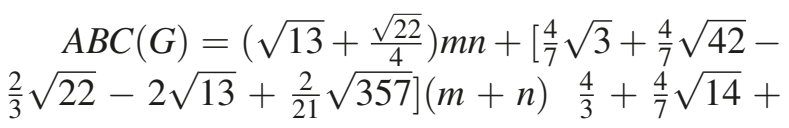


$\frac{4}{5} \sqrt{10}+4 \sqrt{13}-\frac{10}{21} \sqrt{357}-\frac{20}{7} \sqrt{3}-\frac{40}{21} \sqrt{42}+$ $\frac{7}{4} \sqrt{22}+1$.

Theorem 5.2 Let $G \cong R T S(m, s)$. Then, for $m, n \geq$ 3 , the $G A(A)$ is

$G A(G)=\left[\frac{39}{5}\right] m n+\left[\frac{6}{5} \sqrt{21}+\frac{16}{19} \sqrt{21}-\frac{48}{5}-\right.$ $6] m+\left[\frac{6}{5} \sqrt{21}+\frac{16}{19} \sqrt{21}-\frac{48}{5}-6\right] n+\frac{2}{3} \sqrt{35}+$ $\frac{25}{17} \sqrt{15}-\frac{80}{19} \sqrt{21}-4 \sqrt{21}+\frac{161}{5}$.

Proof. Put the information given in Table 7 in $G A(G)=\sum_{\mu v E(G)} 2 \frac{\sqrt{d_{u} d_{v}}}{\left(d_{u}+d_{v}\right)}$, we have

$$
G A(G)=2 \times 2 \sqrt{\frac{3 \times 3}{3+3}}+4 \times 2 \sqrt{\frac{5 \times 7}{5+7}}+2 \times
$$

$2 \sqrt{\frac{5 \times 12}{5+12}}+4 \times 2 \sqrt{\frac{3 \times 5}{3+5}}+(6 m+6 n-20) \times 2 \sqrt{\frac{3 \times 7}{3+7}}$

$+(6 m n-12 m-12 n+24) \times 2 \sqrt{\frac{3 \times 12}{3+12}}+(2 m+2 n-$

10) $\times 2 \sqrt{\frac{7 \times 7}{7+7}}+(4 m+4 n-20) \times 2 \sqrt{\frac{7 \times 12}{7+12}}+(3 m n-$ $8 m-8 n+21) \times 2 \sqrt{\frac{12 \times 12}{12+12}}$.

After simplification, we get

$G A(G)=\left[\frac{39}{5}\right] m n+\left[\frac{6}{5} \sqrt{21}+\frac{16}{19} \sqrt{21}-\frac{48}{5}-\right.$ $6] m+\left[\frac{6}{5} \sqrt{21}+\frac{16}{19} \sqrt{21}-\frac{48}{5}-6\right] n+\frac{2}{3} \sqrt{35}+$ $\frac{25}{17} \sqrt{15}-\frac{80}{19} \sqrt{21}-4 \sqrt{21}+\frac{161}{5}$.

In the table below, we give the edge partition of a graph $G \cong R T S(m, s)$ based on vertices degree sum which are located at unit distance from the final vertices of each edge.

In the next two results, we calculate the $A B C_{4}$ and $G A_{5}$ indices for a graph $G$ of rectangular tetra sheet $\operatorname{RTS}(m, n)$.

Theorem 5.3 For $m, n \geq 6$, the formula for $A B C_{4}$ index of the graph $G \cong R T S(m, n)$ is

$A B C_{4}(G)=\left[\frac{\sqrt{310}}{15}+\frac{\sqrt{178}}{30}\right] m n+\left[\frac{2}{611} \sqrt{876762}+\right.$ $\frac{2}{65} \sqrt{845}+\frac{4}{1457} \sqrt{27683}+\frac{\sqrt{16895}}{155}+\frac{\sqrt{570}}{20}-$ $\frac{4}{15} \sqrt{310}+\frac{2}{47} \sqrt{92}+\frac{5}{47} \sqrt{47}+\frac{2}{15} \sqrt{21}-$ $\left.\frac{7}{30} \sqrt{178}\right](m+n)+\frac{8}{17} \sqrt{2}+\frac{4}{323} \sqrt{34328}+$ $\frac{\sqrt{74}}{19}+\frac{2}{893} \sqrt{148238}+\frac{2}{665} \sqrt{70490}+\frac{3}{4} \sqrt{2}+$ $\frac{\sqrt{2010}}{45}+\frac{\sqrt{41610}}{219}+\frac{\sqrt{30}}{6}+\frac{\sqrt{15038}}{292}+\frac{4}{247} \sqrt{15314}+$ $\frac{2}{295} \sqrt{26910}-\frac{16}{611} \sqrt{86762}+\frac{6}{455} \sqrt{42770}-$ $\frac{4}{65} \sqrt{845}+\frac{4}{465} \sqrt{11470}-\frac{32}{1457} \sqrt{27683}+\frac{6}{1085} \sqrt{23870}+$ $\frac{4}{2263} \sqrt{230826}-\frac{8}{155} \sqrt{16895}+\frac{2}{105} \sqrt{455}+$ $\frac{2}{219} \sqrt{7811}-\frac{2}{5} \sqrt{570}+\frac{16}{15} \sqrt{310}+\frac{4}{47} \sqrt{94}+$ $\frac{8}{1095} \sqrt{10585}+\frac{\sqrt{123}}{15}-\frac{20}{47} \sqrt{92}+\frac{2}{329} \sqrt{15134}-$ $\frac{45}{47} \sqrt{47}+\frac{\sqrt{518}}{35}+\frac{\sqrt{55115}}{365}+\frac{\sqrt{117530}}{730}-\frac{9}{40} \sqrt{158}-$ $\frac{6}{5} \sqrt{21}+\frac{13}{18} \sqrt{178}$.
Table 8. Table 8: Edge Partition of a graph $\mathrm{G} \cong$ $\operatorname{RTS}(m, n)$ based on vertices degree sum which are located at unit distance from the final vertices of each edge.

\begin{tabular}{|c|c|}
\hline $\begin{array}{c}\left(S_{u}, S_{v}\right) \text { where } \\
u v \in E(G)\end{array}$ & Number of edges \\
\hline$(17,17)$ & 2 \\
\hline$(17,38)$ & 8 \\
\hline$(38,38)$ & 2 \\
\hline$(38,47)$ & 4 \\
\hline$(38,70)$ & 4 \\
\hline$(24,32)$ & 4 \\
\hline$(24,45)$ & 4 \\
\hline$(24,73)$ & 4 \\
\hline$(32,45)$ & 4 \\
\hline$(32,73)$ & 2 \\
\hline$(26,38)$ & 8 \\
\hline$(26,45)$ & 4 \\
\hline$(26,47)$ & $4 m+4 n-32$ \\
\hline$(26,70)$ & 6 \\
\hline$(26,80)$ & $2 m+2 n-16$ \\
\hline$(31,45)$ & 4 \\
\hline$(31,47)$ & $2 m+2 n-16$ \\
\hline$(31,70)$ & 4 \\
\hline$(31,73)$ & 4 \\
\hline$(31,80)$ & $4 m+4 n-32$ \\
\hline$(36,70)$ & 2 \\
\hline$(36,73)$ & 4 \\
\hline$(36,80)$ & $6 m+6 n-48$ \\
\hline$(36,90)$ & $6 m n-24 m-24 n+96$ \\
\hline$(45,47)$ & 4 \\
\hline$(45,73)$ & 4 \\
\hline$(45,80)$ & 4 \\
\hline$(47,47)$ & $2 m+2 n-20$ \\
\hline$(47,70)$ & 4 \\
\hline$(47,80)$ & $4 m+4 n-36$ \\
\hline$(70,80)$ & 4 \\
\hline$(73,80)$ & $2 m+2 n-18$ \\
\hline$(73,90)$ & $4 m+4 n-36$ \\
\hline$(80,80)$ & \\
\hline$(80,90)$ & \\
\hline$(90,90)$ & $3 m n-14 m-14 n+65$ \\
\hline & \\
\hline
\end{tabular}


Proof. Based on the information given in Table 8, we compute the $A B C_{4}$ index of $G$ as follows

$$
\begin{aligned}
& A B C_{4}(G)=\sum_{u v \in E(G)} \sqrt{\frac{S_{u}+S_{v}-2}{S_{u} S_{v}}}=2 \sqrt{\frac{17+17-2}{17 \times 17}}+ \\
& 8 \sqrt{\frac{17+38-2}{17 \times 38}}+2 \sqrt{\frac{38+38-2}{38 \times 38}}+4 \sqrt{\frac{24+32-2}{24 \times 32}}+ \\
& 4 \sqrt{\frac{24+45-2}{24 \times 45}}+4 \sqrt{\frac{\frac{32+45-2}{32 \times 45}}{4}}+4 \sqrt{\frac{45+47-2}{45 \times 47}}+ \\
& 4 \sqrt{\frac{47+38-2}{47 \times 38}}+8 \sqrt{\frac{26+38-2}{26 \times 38}}+4 \sqrt{\frac{26+45-2}{26 \times 45}}+ \\
& 4 \sqrt{\frac{38+70-2}{38 \times 70}}+4 \sqrt{\frac{24+73-2}{24 \times 73}}+2 \sqrt{\frac{32+73-2}{32 \times 73}}+(4 m+ \\
& 4 n-32) \sqrt{\frac{26+47-2}{26 \times 47}}+6 \sqrt{\frac{26+70-2}{26 \times 70}}+(2 m+2 n- \\
& \text { 16) } \sqrt{\frac{26+80-2}{26 \times 80}}+4 \sqrt{\frac{31+45-2}{31 \times 45}}+(2 m+2 n- \\
& \text { 16) } \sqrt{\frac{31+47-2}{31 \times 47}}+4 \sqrt{\frac{31+70-2}{31 \times 70}}+4 \sqrt{\frac{31+73-2}{31 \times 73}}+(4 m+ \\
& 4 n-32) \sqrt{\frac{31+80-2}{31 \times 80}}+2 \sqrt{\frac{36+70-2}{36 \times 70}}+4 \sqrt{\frac{36+73-2}{36 \times 73}}+ \\
& (6 m+6 n-48) \sqrt{\frac{36+80-2}{36 \times 80}}+(6 m n-24 m-24 n+ \\
& \text { 96) } \sqrt{\frac{36+90-2}{36 \times 90}}+4 \sqrt{\frac{45+73-2}{45 \times 73}}+4 \sqrt{\frac{45+80-2}{45 \times 80}}+ \\
& +(2 m+2 n-20) \sqrt{\frac{47+47-2}{47 \times 47}}+4 \sqrt{\frac{47+70-2}{47 \times 70}}+(4 m+ \\
& 4 n-36) \sqrt{\frac{47+80-2}{47 \times 80}}+4 \sqrt{\frac{70+80-2}{70 \times 80}}+4 \sqrt{\frac{73+80-2}{73 \times 80}}+ \\
& 2 \sqrt{\frac{73+90-2}{73 \times 90}}+(2 m+2 n-18) \sqrt{\frac{80+80-2}{80 \times 80}}+(4 m+ \\
& 4 n-36) \sqrt{\frac{80+90-2}{80 \times 90}}+(3 m n-14 m-14 n+ \\
& \text { 65) } \sqrt{\frac{90+90-2}{90 \times 90}} \text {. }
\end{aligned}
$$

Further simplification give us the required result $A B C_{4}(G)=\left[\frac{\sqrt{310}}{15}+\frac{\sqrt{178}}{30}\right] m n+$ $\left[\frac{2}{611} \sqrt{876762}+\frac{2}{65} \sqrt{845}+\frac{4}{1457} \sqrt{27683}+\frac{\sqrt{16895}}{155}+\right.$ $\frac{\sqrt{570}}{20}-\frac{4}{15} \sqrt{310}+\frac{2}{47} \sqrt{92}+\frac{5}{47} \sqrt{47}+\frac{2}{15} \sqrt{21}-$ $\left.\frac{7}{30} \sqrt{178}\right](m+n)+\frac{8}{17} \sqrt{2}+\frac{4}{323} \sqrt{34328}+$ $\frac{\sqrt{74}}{19}+\frac{2}{893} \sqrt{148238}+\frac{2}{665} \sqrt{70490}+\frac{3}{4} \sqrt{2}+$ $\frac{\sqrt{2010}}{45}+\frac{\sqrt{41610}}{219}+\frac{\sqrt{30}}{6}+\frac{\sqrt{15038}}{292}+\frac{4}{247} \sqrt{15314}+$ $\frac{2}{295} \sqrt{26910}-\frac{16}{611} \sqrt{86762}+\frac{6}{455} \sqrt{42770}-$ $\frac{4}{65} \sqrt{845}+\frac{4}{465} \sqrt{11470}-\frac{32}{1457} \sqrt{27683}+\frac{6}{1085} \sqrt{23870}+$ $\frac{4}{2263} \sqrt{230826}-\frac{8}{155} \sqrt{16895}+\frac{2}{105} \sqrt{455}+$ $\frac{2}{219} \sqrt{7811}-\frac{2}{5} \sqrt{570}+\frac{16}{15} \sqrt{310}+\frac{4}{47} \sqrt{94}+$ $\frac{8}{1095} \sqrt{10585}+\frac{\sqrt{123}}{15}-\frac{20}{47} \sqrt{92}+\frac{2}{329} \sqrt{15134}-$ $\frac{45}{47} \sqrt{47}+\frac{\sqrt{518}}{35}+\frac{\sqrt{55115}}{365}+\frac{\sqrt{117530}}{730}-\frac{9}{40} \sqrt{158}-$ $\frac{6}{5} \sqrt{21}+\frac{13}{18} \sqrt{178}$.

Theorem 5.4 For $m, n \geq 6$, the $G A_{5}$ index of a graph $G \cong R T S(m, n)$ is

$$
G A_{5}(G)=\left[\frac{12}{7} \sqrt{10}+3\right] m n+\left[\frac{8}{73} \sqrt{1222}+\right.
$$
$\frac{8}{53} \sqrt{65}+\frac{72}{29} \sqrt{5}-\frac{48}{7} \sqrt{10}+\frac{32}{127} \sqrt{235}+\frac{48}{17} \sqrt{2}+$ $\left.\frac{2}{39} \sqrt{1457}+\frac{32}{111} \sqrt{155}-11\right](m+n)+\frac{16}{55} \sqrt{646}+$ $\frac{8}{85} \sqrt{1786}+\frac{96}{77} \sqrt{10}+\frac{16}{105} \sqrt{146}+\frac{16}{7} \sqrt{3}+\frac{16}{23} \sqrt{30}+$ $\frac{\sqrt{247}}{2}+\frac{8}{71} \sqrt{1170}-\frac{64}{73} \sqrt{1222}+\frac{\sqrt{455}}{4}-\frac{64}{53} \sqrt{65}+$ $\frac{6}{19} \sqrt{155}+\frac{12}{53} \sqrt{70}+\frac{48}{109} \sqrt{73}-\frac{576}{29} \sqrt{5}+\frac{192}{7} \sqrt{10}+$ $\frac{6}{23} \sqrt{235}+\frac{12}{59} \sqrt{365}+\frac{8}{117} \sqrt{3290}-\frac{288}{127} \sqrt{235}+$ $\frac{16}{17} \sqrt{14}+\frac{32}{153} \sqrt{365}+\frac{12}{163} \sqrt{730}-\frac{432}{17} \sqrt{2}+\frac{96}{77} \sqrt{10}+$ $\frac{32}{97} \sqrt{438}-\frac{16}{39} \sqrt{1457}+\frac{8}{101} \sqrt{2170}+\frac{\sqrt{2263}}{13}-$ $\frac{256}{111} \sqrt{155}+\frac{96}{25}+61$.

Proof. By following the instructions about the edge partitioning in Table 8, we compute the $G A_{5}$ index of the graph $G$ as follows

$$
\begin{aligned}
& G A_{5}(G)=\frac{2 \sqrt{S_{u} S_{v}}}{\left(S_{u}+S_{v}\right)}=\frac{2 \sqrt{17 \times 17}}{17+17} \times 2+\frac{2 \sqrt{17 \times 38}}{17+38} \times \\
& (8)+\frac{2 \sqrt{38 \times 38}}{38+38} \times 2+\frac{2 \sqrt{38 \times 47}}{38+47} \times 4+\frac{2 \sqrt{38 \times 70}}{38+70} \times \\
& 4+\frac{2 \sqrt{24 \times 32}}{24+32} \times 4+\frac{2 \sqrt{24 \times 45}}{24+45} \times 4+\frac{2 \sqrt{24 \times 73}}{24+73} \times \\
& 4+\frac{2 \sqrt{32 \times 45}}{32+45} \times 4+\frac{2 \sqrt{32 \times 73}}{32+73} \times 2+\frac{2 \sqrt{26 \times 38}}{26+38} \times \\
& 8+\frac{2 \sqrt{26 \times 45}}{26+45} \times 4+\frac{2 \sqrt{26 \times 47}}{26+47} \times(4 m+4 n-32)+ \\
& \frac{2 \sqrt{26 \times 70}}{26+70} \times 6+\frac{2 \sqrt{26 \times 80}}{26+80} \times(2 m+2 n-16)+ \\
& \frac{2 \sqrt{31 \times 45}}{31+45} \times 4+\frac{2 \sqrt{31 \times 47}}{31+47} \times(2 m+2 n-16)+ \\
& \frac{2 \sqrt{31 \times 70}}{31+70} \times 4+\frac{2 \sqrt{31 \times 73}}{31+73} \times 4+\frac{2 \sqrt{31 \times 80}}{31+80} \times(4 m+ \\
& 4 n-32)+\frac{2 \sqrt{36 \times 70}}{36+70} \times 2+\frac{2 \sqrt{36 \times 73}}{36+73} \times 4+\frac{2 \sqrt{36 \times 80}}{36+80} \times \\
& (6 m+6 n-48)+\frac{2 \sqrt{36 \times 90}}{36+90} \times(6 m n-24 m- \\
& 24 n+96)+\frac{2 \sqrt{45 \times 47}}{45+47} \times 4+\frac{2 \sqrt{45 \times 73}}{45+73 \times 4}+\frac{2 \sqrt{45 \times 80}}{45+80} \times \\
& \left.4+\frac{2 \sqrt{47 \times 47}}{47+47} \times\right)(2 m+2 n-20)+\frac{2 \sqrt{47 \times 70}}{47+70} \times 4+ \\
& \frac{2 \sqrt{47 \times 80}}{47+80} \times(4 m+4 n-36)+\frac{2 \sqrt{70 \times 80}}{70+80} \times 4+ \\
& \frac{2 \sqrt{73 \times 80}}{73+80} \times 4+\frac{2 \sqrt{73 \times 90}}{73+90} \times 2+\frac{2 \sqrt{80 \times 80}}{80+80} \times(2 m+2 n- \\
& 18)+\frac{2 \sqrt{80 \times 90}}{80+90} \times(4 m+4 n-36)+\frac{2 \sqrt{90 \times 90}}{90+9} \times(3 m n- \\
& 14 m-14 n+65) .
\end{aligned}
$$

Further simplification give us the required result

$G A_{5}(G)=\left[\frac{12}{7} \sqrt{10}+3\right] m n+\left[\frac{8}{73} \sqrt{1222}+\right.$ $\frac{8}{53} \sqrt{65}+\frac{72}{29} \sqrt{5}-\frac{48}{7} \sqrt{10}+\frac{32}{127} \sqrt{235}+\frac{48}{17} \sqrt{2}+$ $\left.\frac{2}{39} \sqrt{1457}+\frac{32}{111} \sqrt{155}-11\right](m+n)+\frac{16}{55} \sqrt{646}+$ $\frac{8}{85} \sqrt{1786}+\frac{96}{77} \sqrt{10}+\frac{16}{105} \sqrt{146}+\frac{16}{7} \sqrt{3}+\frac{16}{23} \sqrt{30}+$ $\frac{\sqrt{247}}{2}+\frac{8}{71} \sqrt{1170}-\frac{64}{73} \sqrt{1222}+\frac{\sqrt{455}}{4}-\frac{64}{53} \sqrt{65}+$ $\frac{6}{19} \sqrt{155}+\frac{12}{53} \sqrt{70}+\frac{48}{109} \sqrt{73}-\frac{576}{29} \sqrt{5}+\frac{192}{7} \sqrt{10}+$ $\frac{6}{23} \sqrt{235}+\frac{12}{59} \sqrt{365}+\frac{8}{117} \sqrt{3290}-\frac{288}{127} \sqrt{235}+$ $\frac{16}{17} \sqrt{14}+\frac{32}{153} \sqrt{365}+\frac{12}{163} \sqrt{730}-\frac{432}{17} \sqrt{2}+\frac{96}{77} \sqrt{10}+$ $\frac{32}{97} \sqrt{438}-\frac{16}{39} \sqrt{1457}+\frac{8}{101} \sqrt{2170}+\frac{\sqrt{2263}}{13}-$ $\frac{256}{111} \sqrt{155}+\frac{96}{25}+61$.

\section{Conclusion}

In this article, we have done computation of some degree based topological indices for certain networks sheets. As a consequence, we got formu- 
las for these networks. We hope this will help people in the field of network science understand and explore the basic topology of these chemical networks.

For future work, we plan or study the design of some new architectures / networks and their topological properties which play an important role to understand their underlying topologies.

\section{Acknowledgment}

This project was funded by the Deanship of Scientific Research (DSR) at King Abdulaziz University, Jeddah under grant no. (RG-19-135-38). The authors, therefore, acknowledge with thanks DSR for technical and financial support.

\section{References}

[1] S. Alikhani1, R. Hasni, N. E. Arif, On the AtomBond Connectivity Index of Some Families of Dendrimers, J. Comput. Theor. Nanosci. 11(2014), $1-4$.

[2] M. Bac̆a, J. Horva $a^{\prime}$ thova $a^{\prime}$, M. Mokrišova', A. Suh $a^{\prime}$ nyiov $\breve{a}$, On topological indices of fullerenes, Appl. Math. Comput. 251(2015), 154-161.

[3] A. Q. Baig, M. Imran, H. Ali, Computing Omega, Sadhana and PI polynomials of benzoid carbon nanotubes, Optoelectron. Adv. Mater. Rapid Communin. 9(2015), $248-255$.

[4] A. Q. Baig, M. Imran, H. Ali, On Topological Indices of Poly Oxide, Poly Silicate, DOX and DSL Networks, Canad. J. Chem. Accepted, in press.

[5] M. Deza, P. W. Fowler, A. Rassat, K. M. Rogers, Fullerenes as tiling of surfaces, J. Chem. Inf. Comput. Sci. 40(2000), P550 - 558.

[6] M. V. Diudea, I. Gutman, J. Lorentz, Molecular Topology, Nova, Huntington, 2001.

[7] E. Estrada, L. Torres, L. Rodrez, I. Gutman, An atom-bond connectivity index: Modelling the enthalpy of formation of alkanes, Indian J. Chem. $37 A(1998), 849-855$.

[8] W. Gao, W. Wang, M. R. Farahani, Topological indices study of molecular structure in anticancer drugs, J. Chem. vol. 2016, Article ID 3216327, 8 pages, 2016.

[9] M. Ghorbani, M. A. Hosseinzadeh, Computing $A B C_{4}$ index of nanostar dendrimers, Optoelectron. Adv. Mater. Rapid Commun. 4(2010) , 1419 1422.
[10] A. Graovac, M. Ghorbani, M. A. Hosseinzadeh, Computing fifth geometric-arithmetic index for nanostar dendrimers, J. Math. Nanosci. 1(2011), $33-42$.

[11] I. Gutman, O. E. Polansky, Mathematical concepts in organic chemistry, Springer-Verlag, New York, 1986.

[12] S. Hayat, M. Imran, Computation of certain topological indices of nanotubes, J. Comput. Theor. Nanosci. 12(2015), $70-76$.

[13] S. Hayat, M. Imran, Computation of certain topological indices of nanotubes covered by $C_{5}$ and $C_{7}$, J. Comput. Theor. Nanosci., 12(2015), 533 - 541.

[14] S. Hayat, M. Imran, On some degree based topological indices of certain nanotubes, J. Comput. Theor. Nanosci. Accepted, in press.

[15] S. Hayat, M. Imran, Computation of topological indices of certain networks, Appl. Math. Comput. 240(2014), $213-228$.

[16] A. Iranmanesh, M. Zeraatkar, Computing GA index for some nanotubes, Optoelectron. Adv. Mater. Rapid Commun. 4(2010), 1852 - 1855.

[17] W. Lin, J. Chen, Q. Chen, T. Gao, X. Lin, B. Cai, Fast computer search for trees with minimal ABC index based on tree degree sequences, MATCH Commun. Math. Comput. Chem. 72(2014), $699-$ 708.

[18] P. D. Manuel, M. I. Abd-El-Barr, I. Rajasingh, B. Rajan, An efficient representation of Benes networks and its applications, J. Discrete Algorithms, 6(2008), $11-19$.

[19] S. Manzoor, M. K. Siddiqui, S. Ahmad, On entropy measures of molecular graphs using topological indices, Arab. J. Chem. 13(2020), 6285 - 6298.

[20] M. Nadeem, A. Yousaf, H. Alolaiyan, A. Razaq, Certain polynomials and related topological indices for the series of benzenoid graphs, Sci Rep.9,9129 (2019). https://doi.org/10.1038/s41598-019-45721-y.

[21] J. L. Palacios, A resistive upper bound for the ABC index, MATCH Commun. Math. Comput. Chem. 72(2014), $709-713$.

[22] M. Randic', On Characterization of molecular branching, J. Amer. Chem. Soc., 97(1975), 66096615.

[23] D. Vukičevic $c^{\prime}$ B. Furtula, Topological index based on the ratios of geometrical and arithmetical means of end-vertex degrees of edges, J. Math. Chem., 46(2009), $1369-1376$.

[24] H. Wiener, Structural determination of paraffin boiling points, J. Amer. Chem. Soc., 69(1947), $17-20$. 


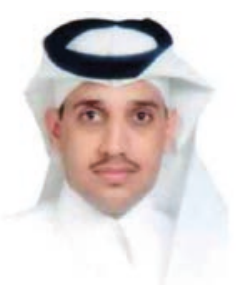

Dr. Fawaz E. Alsaadi received the B.S. and M.Sc. degrees in computer science from King Abdulaziz University, Jeddah, Saudi Arabia, and University of Denver, Denver, Colorado, USA, respectively. He received the Ph.D. degree in biometric security from the University of Colorado Springs, Colorado Springs, Colorado, USA. He is currently an assistant professor of the Information Technology Department within the Faculty of Computing and Information Technology, King Abdulaziz University, Jeddah, Saudi Arabia. He has research interests in biometric recognition and biometric security, information security, and cloud computing storage security.

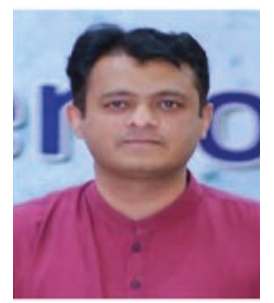

Dr. Syed Ahtsham Ul haq Bokhary was born in Hafizabad, Pakistan in 1981. He received the M. Sc Mathematics degree in 2003 from Punjab University, Lahore, Pakistan , MS Mathematics in 2005 from Quaid-IAzam University, Islamabad, Pakistan and Ph.D. degree in 2010 from Abdus Salam school of Mathematical Sciences, GCU, Lahore, Pakistan. From 2012, he is serving as Assistant Professor of Mathematics in Centre of Advance studies in Pure and Applied Mathematics, Bahauddin Zakaiya University, Multan, Pakistan. He is the author of more than 35 articles. His research interest is mainly in the area of Graph theory and Combinatorics. More specifically, His research interest are in Coloring of graphs and hypergraphs, graph labeling, distances in graphs, algebraic graph theory, molecular graph theory, spectral graph theory and extremal graph theory.

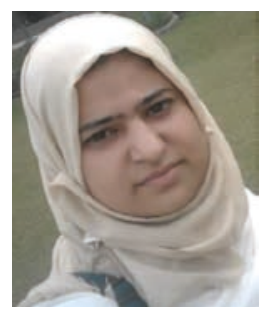

Miss. Aqsa Shah received her M.Sc. in Mathematics from Bahauddin Zakariya University, Multan in 2014 and Mphil Mathematics from BZU, Multan in 2016 and currently enrolled in Centre of Advanced Studies in Pure and Applied Mathematics, CASPAM, BZU, Multan as a Ph.D. scholar session 2018-2022. She is currently Ph.D. scholar at Bahauddin Zakariya University and has research interests in combinatorics, graph theory, and control theory.

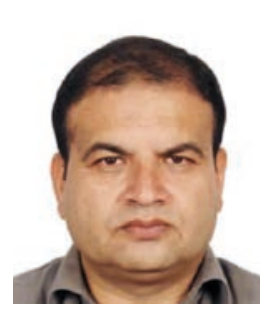

Dr. Usman Ali received his M.Sc. in Mathematics from University of Peshawar in 2003 and Ph.D. in mathematics from Abdus Salam School of Mathematical Sciences GC University Lahore in 2009. He is currently an associate professor at Bahauddin Zakariya University and has research interests in combinatorics, graphs, braids, and knots; and the interplay among these mathematical notions. Apart from mathematics, he likes to different sports, including cricket and soccer.

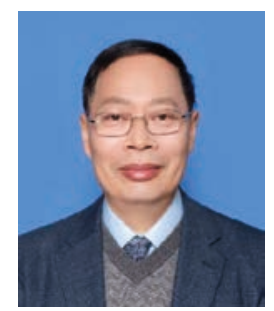

Dr. Jinde Cao (Fellow, IEEE) received the B.S. degree from Anhui Normal University, Wuhu, China, the M.S. degree from Yunnan University, Kunming, China, and the Ph.D. degree from Sichuan University, Chengdu, China, all in mathematics/applied mathematics, in 1986, 1989, and 1998, respectively. He is an Endowed Chair Professor, the Dean of the School of Mathematics, the Director of the Jiangsu Provincial Key Laboratory of Networked Collective Intelligence of China and the Director of the Research Center for Complex Systems and Network Sciences at Southeast University. Prof. Cao was a recipient of the National Innovation Award of China, Obada Prize and the Highly Cited Researcher Award in Engineering, Computer Science, and Mathematics by Thomson Reuters/Clarivate Analytics. He is elected as a member of the Academy of Europe, a member of the European Academy of Sciences and Arts, a foreign member of Russian Academy of Natural Sciences, a fellow of Pakistan Academy of Sciences, a fellow of African Academy of Sciences, an IASCYS academician, and a full member of Sigma Xi.

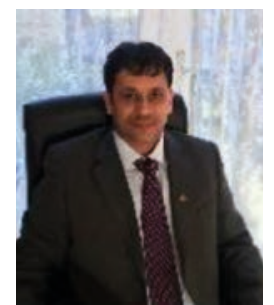

Dr. Madini O. Alassafi received his B.S. degree in Computer Science from King Abdulaziz University, Saudi Arabia in 2006, and received M.S. degree in Computer Science from California Lutheran University, United State of America in 2013. He received the PhD degree in Security Cloud Computing in 2018 from University of Southampton, Southampton, United Kingdom. He is currently work as chairman and as an assistant professor of Information Technology department in the Faculty of Computing and Information Technology at King Abdulaziz University. His research interests are mainly focus on Cloud Computing and Security, Distributed Systems, Internet of Things (IoT) Security issues, Cloud Security Adoption, Risks, Cloud Migration Project Management, Cloud of Things and Security Threats. He has published numerous Conference Papers, Journal Papers and book chapters.

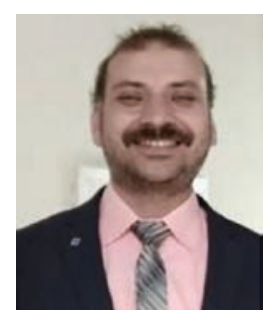

Dr. M. U. Rehman received his M.Sc. in Mathematics from Kohat University of Science and Technology, Pakistan in 2009, MS in Mathematical Modeling and Scintific Computing from Air University Islamabad, Pakistan in 2013 and Ph.D. in Applied Mathematics from University of Science and Technology of China (USTC), P.R. China, in 2019. He has research interests in Algebraic Combinatorics, Spectral Graph Theory, Algebraic Graph Theory, Chemical Graph Thoery, Algebra and algebraic number theory.

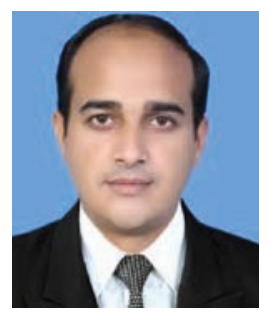

Dr. Jamshaid UI Rahman received his M.S. in Mathematical Modeling and Scintific Computing from Air University Islamabad, Pakistan in 2011 and Ph.D. in Computational Mathematics from the Univesrity of Science and Technology of China, in 2020. His research intrest is modeling and simulation, deep learning and computer vision. 